\title{
Research on Distribution Pattern of Geochemical Field Based on Spatial Auto-/Cross- Correlation Analysis
}

\author{
ZHENGWEN JIANG $^{1}$,BAOYI ZHANG ${ }^{1,2}$, YIRU CHEN ${ }^{1}$ \\ ${ }^{1}$ School of Geosciences \& Info-Physics, Central South \\ University, ChangSha, 410083, \\ jiangzhengwen@csu.edu.cn \\ ${ }^{2}$ MOE Key Laboratory of Metallogenic Prediction of \\ Nonferrous Metals and Geological Environment \\ Monitoring, Central South University, ChangSha, \\ 410083, zhangbaoyi@csu.edu.cn
}

The spatial distribution pattern of the geochemical field is widely used in geological research, environmental protection and mineral prospecting. Geochemical indicators often coexist in complex geological systems with a mutually influential and mutually constrained manner. Therefore, based on the spatial auto-correlation and cross-correlation of geochemical data, the distribution pattern of elements in the geochemical field is explored in this paper. The main work is as follows:

(1) Local spatial auto-correlation indicators are calculated, including Moran' s I、Geary' s C and Getis Ord' s G, which are used to describe the spatial distribution characteristics of elements in the geochemical field.

(2) Exploring the correlation between a pairs of geochemical elements by calculating spatial cross-correlation indicators, including the bivariate global and local Moran's I and the multivariate Geary's C.

(3) According to the univariate local Moran's I clusters results, transforms original geochemical data into geospatial data. Using the Apriori algorithm, spatial association rules between two or more geochemical elements are mined, which provides a new idea for exploring the spatial relationship between geochemical elements, including combinations and patterns of geochemical element.

In summary, the similarity of geochemical element's attribute and location are simultaneously considered in spatial correlation analysis, which is more in line with the complex distribution of geochemical elements in practice in exploring the geochemical field distribution. Traditional spatial correlation indicators can only evaluate the spatial distribution pattern between single element or two elements. The Apriori algorithm Based on spatial autocorrelation in this paper, it is possible to find the combination of elements that frequently appear in the same location, including two or more elements, and also find positive correlation and negative correlation patterns. 